\title{
Geología y geocronología U-Pb del granito de Banabuiú, Noreste de Ceará, Brasil
}

\author{
Geology and U-Pb geochronology of the Banabuiú granite, \\ Northeastern Ceará, Brazil
}

\author{
M.N. Lima1,2, J.A. Nogueira Neto ${ }^{3}$, M.R. Azevedo ${ }^{1,2}$, B. Valle Aguado1,2
}

\begin{abstract}
RESUMEN
El macizo de Banabuiú aflora en el Dominio Ceará Central (DCC) de la Provincia de Borborema (NE Brasil) como una intrusión granítica alargada según la dirección N-S, concordante con las estructuras regionales. Está emplazado en rocas del Paleoproterozoico intensamente deformadas y transformadas en gneises y migmatitas durante la Orogenia Brasiliense ( 600 Ma). Con base en dataciones U-Pb de circones, la edad de cristalización del granito de dos micas de Banabuiú es estimada en 578,6 \pm 6,5 Ma. El granito es fuertemente peraluminoso y presenta una signatura geoquímica de tipo-S. Los valores de $\varepsilon \mathrm{Nd}_{580}$ son marcadamente negativos $\left(\varepsilon \mathrm{Nd}_{580}=-19 \mathrm{a}-23\right)$ y se sobreponen parcialmente con los del Complejo Gnéisico Migmatítico del DCC $\left(\varepsilon \mathrm{Nd}_{580}=-12\right.$ a -26$)$, sugiriendo que el magma parental del granito ha sido generado a través de la fusión parcial de materiales corticales semejantes a los que se encuentran en el DCC.
\end{abstract}

Palabras clave: Orogenia Brasiliense, granitos de dos micas, geocronología U-Pb.

\section{ABSTRACT}

The Banabuiú massif crops out in the Central Ceará Domain (DCC) of the Borborema Province (NE Brasil), as an N-S elongate granite intrusion, concordant with the regional structures. It was emplaced into basement rocks of Paleoproterozoic age, extensively transformed into gneisses and migmatites during the Brasilian orogeny ( 600 Ma). Using U-Pb zircon dating, the crystallization age of the Banabuiú syn-kinematic two-mica granite was estimated at $578.6 \pm 6.5 \mathrm{Ma}$. The granite is strongly peraluminous $(\mathrm{A} / \mathrm{CNK}=1,098-1,134)$ and shows a typical S-type geochemical signature. The $\varepsilon \mathrm{Nd}_{580}$ values are strongly negative $\left(\varepsilon \mathrm{Nd}_{580}=-19 \mathrm{a}-23\right)$ and partially overlap with those of the Paleoproterozoic gneissmigmatite complex $\left(\varepsilon \mathrm{Nd}_{580}=-12\right.$ to -26$)$, suggesting that the parental magmas of the Banabuiú granite could have been produced by partial melting of similar crustal materials.

Key words: Brasilian Orogeny, two-mica granites, U-Pb geochronology.

\section{Introducción}

El granito de Banabuiú es una de las numerosas intrusiones graníticas emplazadas en la Provincia Bororema (PB) durante el final de la Orogenia Brasiliense ( 600 Ma). Los terrenos situados en el sector septentrional de esta Provincia fueron subdivididos por Fetter (1999), Fetter et al. (2000) y Brito Neves et al. (2000) en los siguientes dominios: a) Dominio del NW del Ceará (NWC), b) Dominio del Ceará Central (DCC) y c) Dominio del Rio Grande del Norte (DRGN). Posteriormente, Arthaud et al. (1998) y Arthaud (2005) individualizaron un cuarto dominio, al que designaron Orós-Jaguaribe (DOJ), localizado entre el DCC y el DRGN.

La región de Banabuiú se localiza en el Dominio del Ceará Central (DCC), en el límite con el Dominio Orós Jaguaribe (DOJ). Las principales unidades

\footnotetext{
1 Dep. Geociências da Universidade de Aveiro, 3800 Aveiro, Portugal. Email: marthageolima@yahoo.com.br, mazevedo@ua.pt, baguado@ua.pt

2 Unidad de Investigación GeoBioTec, Portugal

3 Dep. Geologia da Universidade Federal do Ceará, Fortaleza-Ceará-Brasil. Email: nogueira@ufc.br
} 


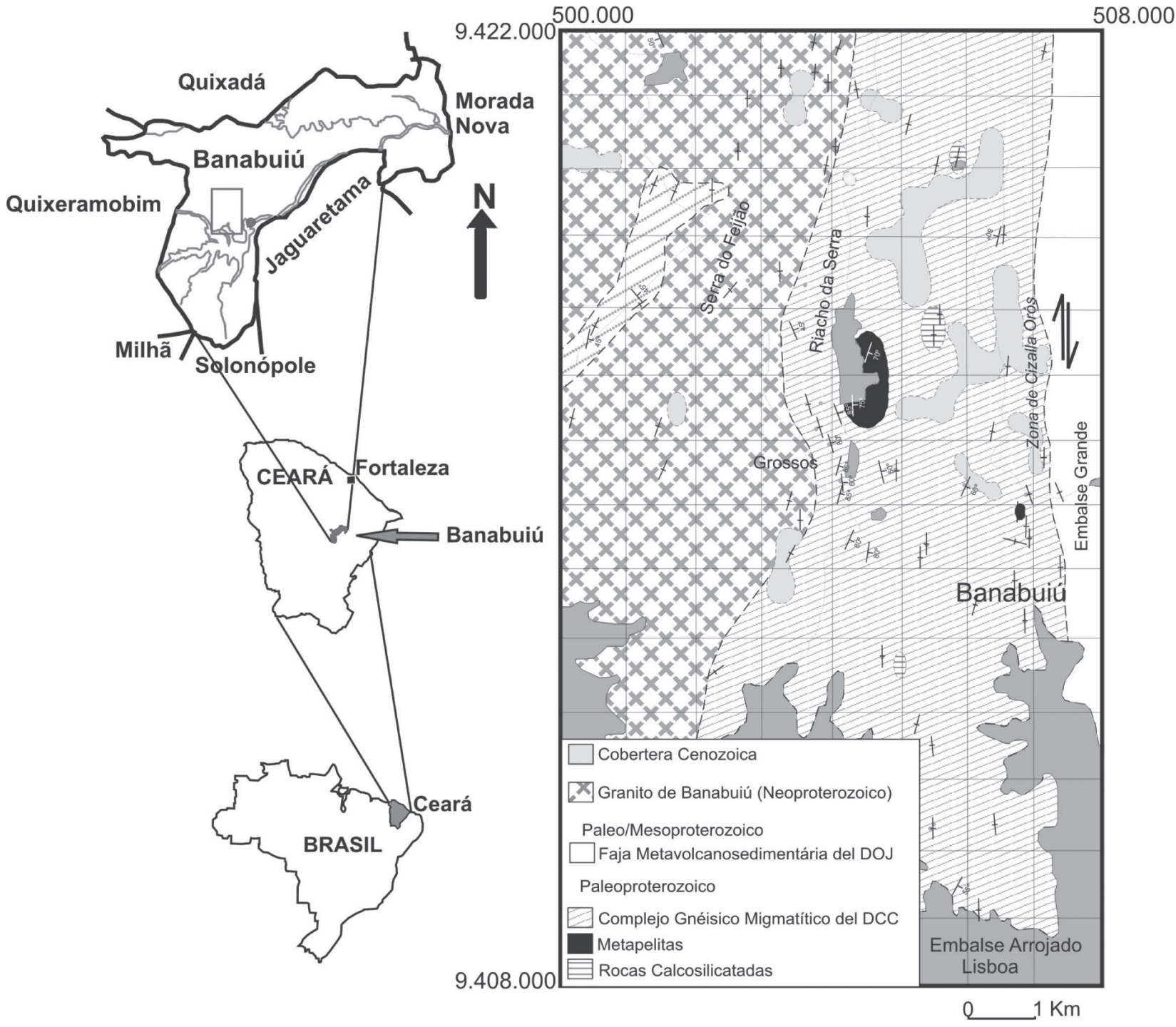

Fig. 1.-Mapa geológico esquemático del área estudiada.

litoestratigráficas en el área son el Complejo Gnéisico Migmatítico del DCC, de edad paleoproterozoica, el granito de Banabuiú (Neoproterozoico), las pegmatitas (Fanerozoico) y, por fin, los depósitos de cobertera aluvionar del Cenozoico (fig. 1).

Durante la Orogenia Brasiliense, que envolvió la convergencia y subsecuente colisión continental de los cratones San Luis-Oeste Africano y San Francisco-Congo-Kasai (Van Schmus et al., 1997), la Provincia Bororema (PB) sufrió deformación y metamorfismo intensos. La actividad tectónica está predominantemente relacionada con el desarrollo de extensas zonas de cizalla transcurrentes, dextras y senestras, y el metamorfismo alcanza la facies anfi- bolítica de grado alto y, localmente, las condiciones de la fusión parcial y producción de migmatitas (Delgado et al., 2003). Las edades U-Pb obtenidas por Fetter (1999) en circones de ortogneises y migmatitas de una de las unidades de la Provincia Borborema permitieron datar sus protolitos con 2,152,10 Ga (Paleoproterozoico medio).

Con base en criterios tectonometamórficos, Arthaud (2005) subdividió los granitoides brasilienses del Ceará en los siguientes grupos: a) pre-colisionales; b) sin-colisionales tangenciales, donde incluye granitos de dos micas tipo-S, formados durante el clímax metamórfico (batolitos de Senador Pompeu y Banabuiú); c) sin-colisionales direccionales, gene- 
rados durante la fase transcurrente con extrusión lateral (batolitos de Quixeramobim y de Quixadá) y d) post-colisionales, asociados al colapso de la cadena brasiliense. Las dataciones U-Pb obtenidas por Nogueira (2004) en circones procedentes de los batolitos graníticos sincinemáticos de Senador Pompeu (561 $\pm 15 \mathrm{Ma})$, Quixadá (585 $\pm 4,7 \mathrm{Ma})$ y Quixeramobim (587 $\pm 14 \mathrm{Ma})$, permiten situar el período de emplazamiento de estos magmas en el intervalo 560-590 Ma. Por otro lado, las edades $\mathrm{Rb}-\mathrm{Sr}$ y $\mathrm{U}-\mathrm{Pb}$ determinadas en rocas intrusivas post-colisionales varían entre 550 y $520 \mathrm{Ma}$ (Tavares Jr, 1992; Fetter, 1999; Matos et al., 2003).

La transición del Neoproterozoico al Paleozoico estuvo además marcada por importantes procesos generadores de cuerpos pegmatíticos. Las dataciones $\mathrm{K}-\mathrm{Ar}$ adquiridas en concentrados de moscovita de las pegmatitas de la región de Banabuiú indican edades de $506 \pm 6,1 \mathrm{Ma}$ (Lima, 2008), coherentes con el intervalo de edades (470-530 Ma) propuesto por Almeida et al. (1968).

\section{El granito de Banabuiú}

El granito de Banabuiú ocupa la parte occidental del área en estudio y constituye un macizo con una forma cartográfica alargada en la dirección N-S, concordante con las estructuras regionales (fig. 1). La facies dominante se caracteriza por una textura homogénea, de grano medio a fino, y por una asociación mineralógica constituida por cuarzo, plagioclasa, feldespato potásico y proporciones semejantes de biotita y moscovita (fig. 2A). Dispersos en estos granitoides se observan con frecuencia «schlieren»

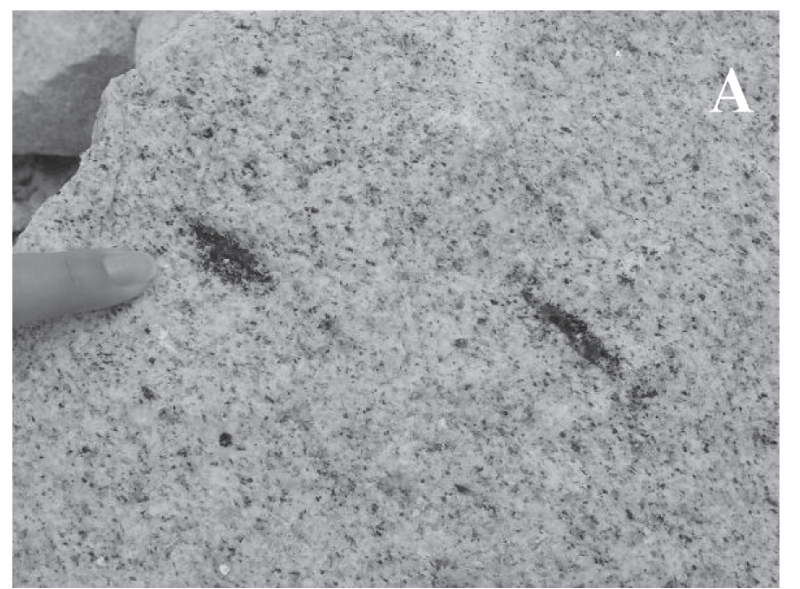

biotíticos y enclaves angulosos de los gneises del encajante metamórfico (fig. 2B). En los bordes oriental y occidental del macizo se desarrolla una foliación subvertical con dirección N-S, generalmente de flujo magmático, aunque en algunos lugares muestra indicios de deformación en el estado sólido, sugiriendo que el emplazamiento del magma ha sido controlado tectónicamente, posiblemente por la Zona Cizalla de Orós (fig. 1). Sin embargo, la ausencia de deformación en la mayor parte del macizo indica que la intrusión es relativamente tardía (Lima, 2008). El macizo de Banabuiú está cortado por numerosos filones de cuarzo, aplitas y pegmatitas con espesor y extensión variables que, en ocasiones, se interceptan.

\section{Petrografía y química mineral}

A escala microscópica, el granito de Banabuiú exhibe textura inequigranular, hipidiomórfica a alotriomórfica, con tamaño de grano fino a medio y contiene como minerales esenciales cuarzo, feldespato potásico, plagioclasa, biotita y moscovita (fig. 3). Circón, apatito y minerales opacos son las fases accesorias más comunes (fig. 3), mientras que sericita y clorita se presentan como productos de la alteración de los minerales primarios. El cuarzo, en granos anhedrales con dimensiones comprendidas entre $0,1 \mathrm{~mm}$ y $3 \mathrm{~mm}$, tiene carácter esencialmente intersticial y la extinción ondulante que a veces presenta indica la poca deformación a que han sido sometidas las rocas. Ocasionalmente aparece como inclusiones redondeadas dentro de cristales de feldespato potásico y plagioclasa.

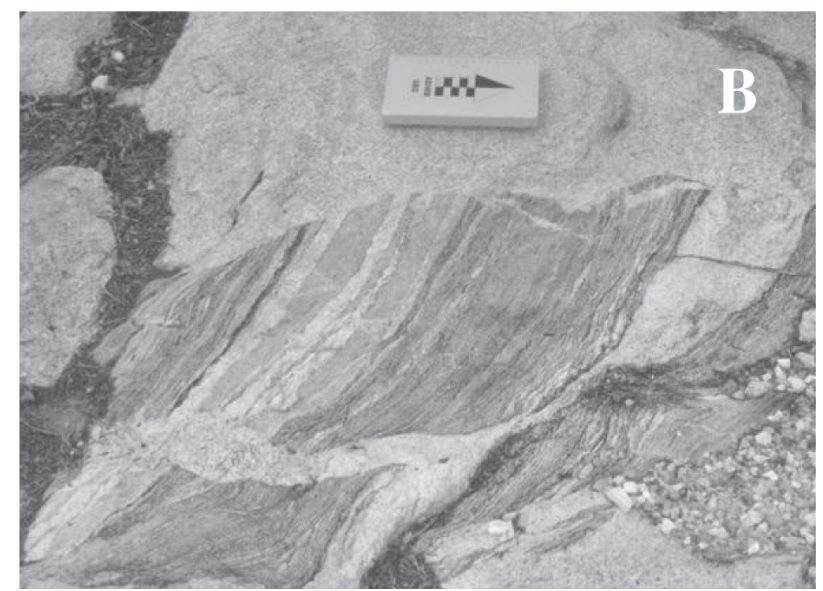

Fig. 2.-Aspectos del granito de Banabuiú en afloramiento: A) «schlieren» biotíticos en el granito de Banabuiú; B) enclaves de gneises del encajante en el granito. 

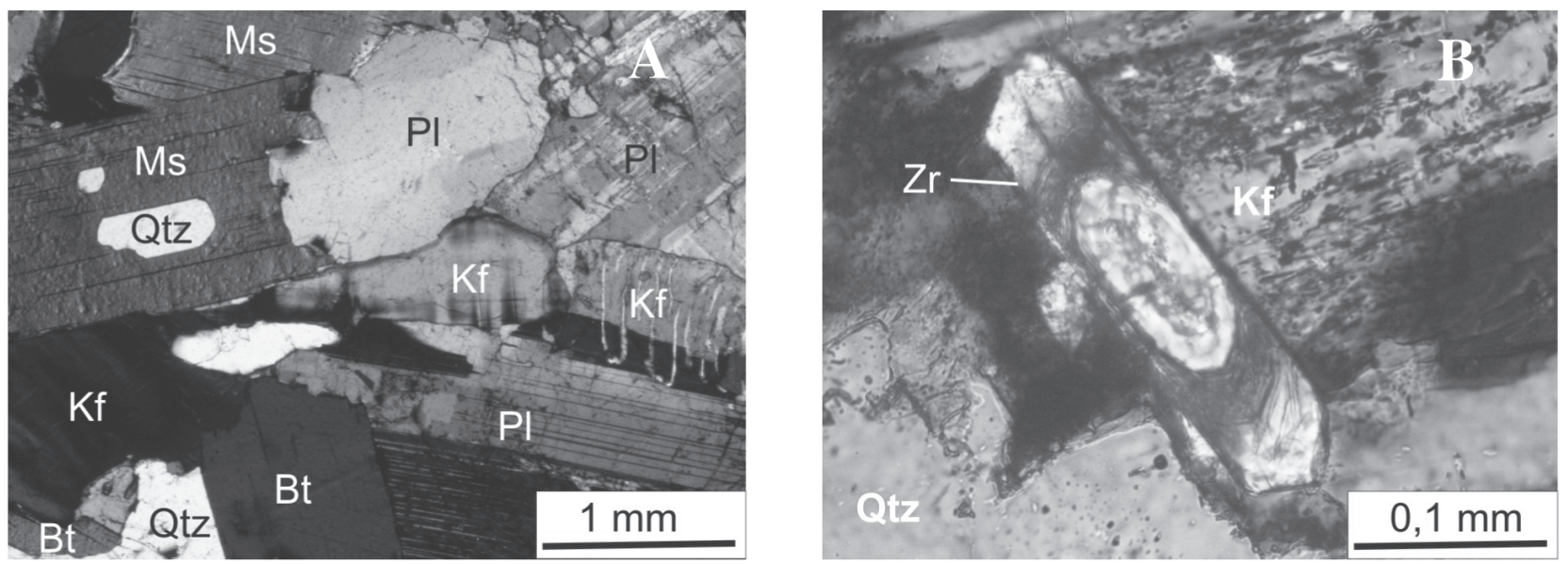

Fig. 3.-Microfotografías del granito de Banabuiú: A) Aspecto general del granito (Qtz: cuarzo, Ms: moscovita, Bt: biotita, PI: plagioclasa, Kf: feldespato potásico); B) Detalle de un cristal idiomorfo de circón en la matriz del granito.

El feldespato potásico constituye, predominantemente, cristales xenomorfos e intersticiales $(0,25-$ $3 \mathrm{~mm})$. Presenta la macla típica de la microclina combinada a veces con la de Carlbad, textura micro- a criptopertítica y composición variable, entre $\mathrm{Or}_{81}$ y $\mathrm{Or}_{94}$. Con frecuencia, en los contactos con la plagioclasa se observan mirmequitas. Además de cuarzo, posee inclusiones de diminutas láminas de biotita $\mathrm{y}$, aunque menos abundantes, también de cristales de plagioclasa. La plagioclasa se presenta en cristales hipidiomorfos y xenomorfos, con dimensiones que varían entre $0,25 \mathrm{~mm}$ y $2,5 \mathrm{~mm}$, encontrándose frecuentemente sericitizada y microfisurada. Muestra la macla polisintética de la albita y zonado óptico poco marcado. En términos químicos, se clasifica como oligoclasa y albita $(\% \mathrm{An}=27-8)$.

La biotita es la principal fase máfica de estas rocas. Aparece como cristales hipidiomorfos, con fuerte pleocroísmo, entre marrón rojizo $(\gamma$ y $\beta)$ y marrón claro $(\alpha)$, y dimensiones que no superan los $2 \mathrm{~mm}$. Químicamente, las biotitas del granito de Banabuiú se proyectan en la zona de transición entre el campo de las biotitas ferríferas y el de las siderofilitas (Foster, 1960). En el diagrama de Nockolds (1947), se sitúan en el campo de las biotitas que coexisten con moscovita, mientras que en el esquema de clasificación de Nachit et al. (1985) muestran afinidades con las series alumino-potásicas, lo que sugiere una procedencia cortical para el granito de Banabuiú (fig. 4). Los cristales de biotita están, con frecuencia, alterados a clorita (pseudoturingita), en particular a lo largo de los planos de exfoliación.
La moscovita primaria se encuentra, en general, como cristales hipidiomorfos $(0,5-2 \mathrm{~mm})$ dispersos en la matriz de la roca. Puede incluir total o parcialmente granos xenomorfos de feldespato potásico y de cuarzo. El circón normalmente se presenta como inclusiones rodeadas de aureolas pleocroicas en cristales de biotita, aunque también se observan pequeños prismas idiomorfos asociados a los otros minerales constituyentes de la roca (fig. 3). El apatito aparece según pequeños cristales incoloros, con relieve alto y color de interferencia gris del primer orden y, al igual que los minerales opacos, está preferentemente asociado a la biotita.

Las características petrográficas del granito de Banabuiú, en particular la presencia en proporciones semejantes de biotita y moscovita, sugieren que el granito deriva de un magma félsico rico en agua y composición peraluminosa, generado en condiciones de anatexia cortical.

\section{Geoquímica}

En el granito de Banabuiú se han llevado a cabo análisis de roca total de 7 muestras, incluyendo elementos mayores, elementos traza y tierras raras. Los análisis completos se muestran en las tablas 1 y 2 .

La proyección de las composiciones normativas CIPW de las muestras del granito de Banabuiú en los diagramas An-Ab-Or (Barker, 1979) y QAP (Le Bas \& Streckeisen, 1991) pone de manifiesto que la mayoría de las muestras analizadas se clasifican como granitos y monzogranitos, respectivamente (fig. 5). 


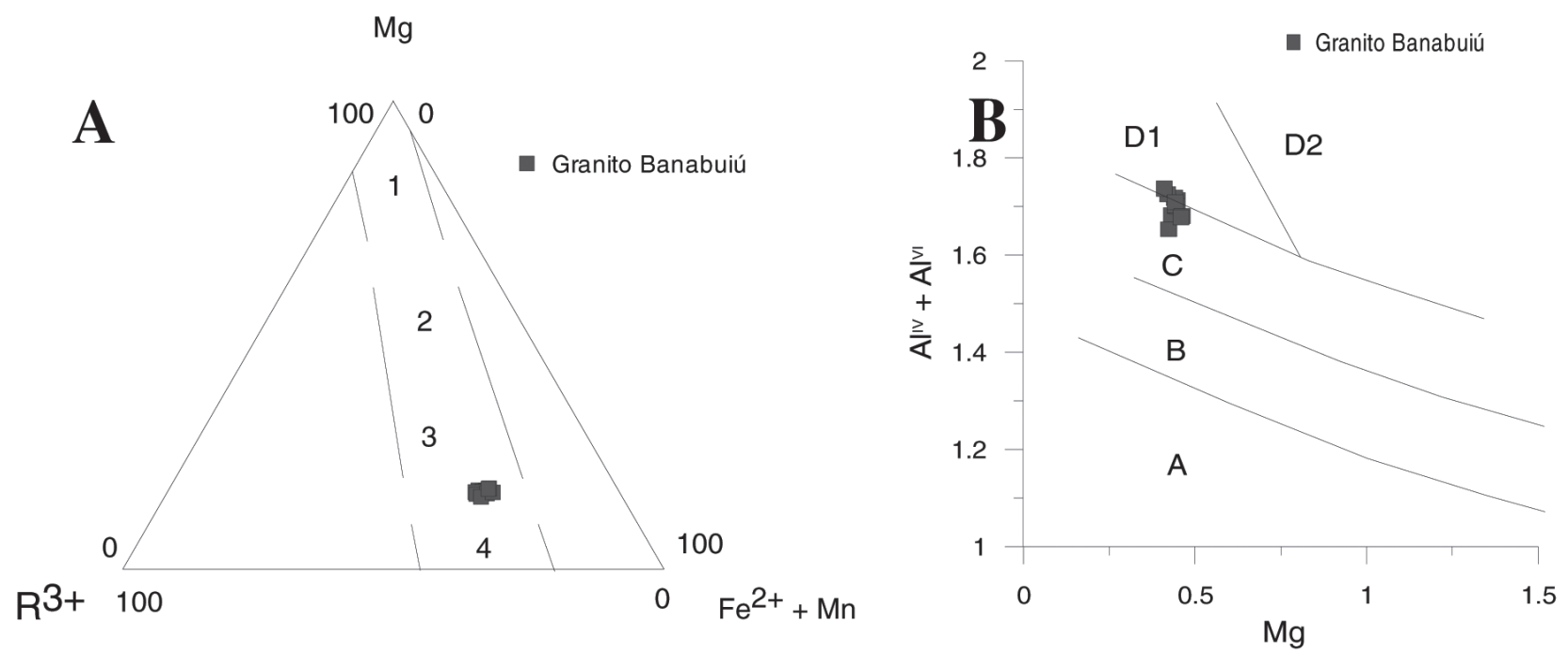

Fig. 4.-A) Representación de la composición de biotitas del granito de Banabuiú en el diagrama de Foster (1960). 1) flogopitas; 2) biotitas magnesianas; 3) biotitas ferríferas; 4) siderofilitas. B) Proyección de las biotitas del granito de Banabuiú en el diagrama Mg versus Al total, según Nachit et al. (1985). A: rocas de series alcalinas; B: series subalcalinas; C: series calco-alcalinas; D1: series alumino-potásicas con biotita \pm moscovita; D2: series alumino-potásicas con biotita \pm cordierita.
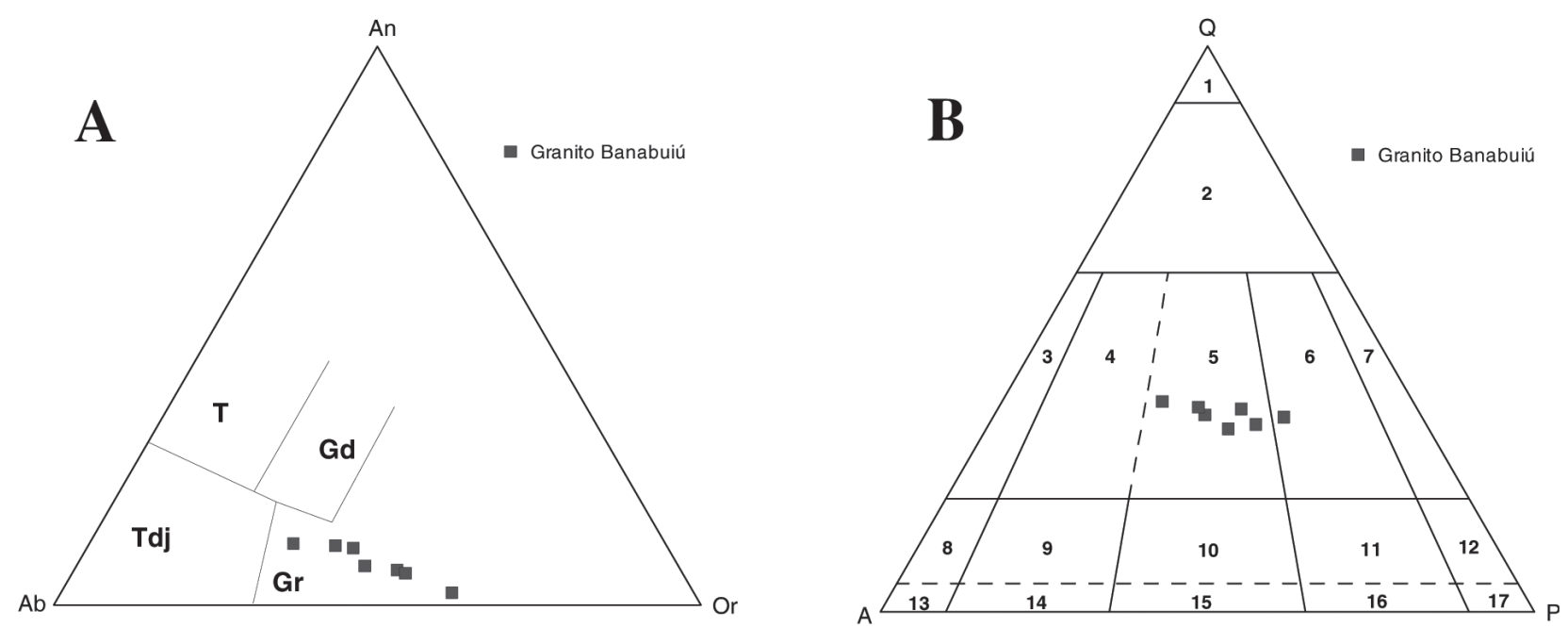

Fig. 5.-Proyección de las muestras del granito de Banabuiú en los diagramas An-Ab-Or (Barker, 1979) y QAP (Le Bas \& Streckeisen, 1991). A) Diagrama An-Ab-Or. T: tonalitas; Gd: granodioritas; Tdj: trondjemitas; Gr: granitos. B) Diagrama QAP (cuarzo-feldespato alcalino-plagioclasa). 1) Cuarzolita; 2) granitoide rico en cuarzo; 3) granito con feldespato alcalino; 4) sienogranito; 5) monzogranito; 6) granodiorita; 7) tonalita; 8) cuarzo-sienita con feldespato alcalino; 9) cuarzo-sienita; 10) cuarzo-monzonita; 11) cuarzo-monzodiorita/cuarzo-monzogabro; 12) cuarzo-diorita/cuarzo-gabro; 13) sienita con feldespato alcalino; 14) sienita; 15) monzonita; 16) monzodiorita/monzogabro; 17) diorita/gabro.

Los elevados valores del índice A/CNK (A/CNK $=1,098-1,134)$ indican un carácter fuertemente peraluminoso, reforzando la hipótesis de una génesis a partir de la fusión parcial de protolitos metasedimentarios. En los diagramas de variación química de elementos mayores, las muestras del granito de Banabuiú definen tendencias coherentes, donde se aprecia disminución de $\mathrm{MgO}, \mathrm{TiO}_{2}, \mathrm{MnO}, \mathrm{CaO}$,
$\mathrm{Na}_{2} \mathrm{O}, \mathrm{P}_{2} \mathrm{O}_{5}$ y aumento de $\mathrm{SiO}_{2}$ y $\mathrm{K}_{2} \mathrm{O}$ cuando disminuye el contenido en $\mathrm{Fe}_{2} \mathrm{O}_{3}$ t (fig. 6). Los contenidos en $\mathrm{Al}_{2} \mathrm{O}_{3}$ también tienden a disminuir cuando aumenta la diferenciación geoquímica, aunque la ausencia de una correlación positiva clara entre $\mathrm{Al}_{2} \mathrm{O}_{3}$ y $\mathrm{Fe}_{2} \mathrm{O}_{3} \mathrm{t}$ sugiere que el fraccionamiento de la plagioclasa podrá no haber sido suficientemente importante como para producir el empobrecimiento 
Tabla 1.-Análisis químicos de elementos mayores $(\%$ peso) y traza $(\mathrm{ppm})$ del granito Banabuiú

\begin{tabular}{|c|c|c|c|c|c|c|c|}
\hline & MI-01 & MI-184 & MI-93A & MI-206 & MI-212 & MI-214 & MI-105G \\
\hline $\mathrm{SiO}_{2}$ & 74,01 & 75,22 & 73,07 & 73,74 & 74,08 & 74,82 & 70,17 \\
\hline $\mathrm{TiO}_{2}$ & 0,12 & 0,12 & 0,27 & 0,21 & 0,12 & 0,14 & 0,34 \\
\hline $\mathrm{Al}_{2} \mathrm{O}_{3}$ & 13,63 & 13,89 & 14,67 & 14,27 & 14,23 & 13,41 & 14,58 \\
\hline $\mathrm{Fe}_{2} \mathrm{O}_{3}(\mathrm{t})$ & 1,35 & 1,27 & 2,41 & 1,64 & 1,34 & 1,38 & 2,85 \\
\hline $\mathrm{MnO}$ & 0,02 & 0,02 & 0,03 & 0,03 & 0,03 & 0,02 & 0,05 \\
\hline $\mathrm{MgO}$ & 0,18 & 0,19 & 0,48 & 0,29 & 0,22 & 0,21 & 0,83 \\
\hline $\mathrm{CaO}$ & 0,88 & 0,82 & 1,55 & 1,33 & 1,05 & 0,52 & 1,74 \\
\hline $\mathrm{Na}_{2} \mathrm{O}$ & 3,17 & 3,07 & 4,12 & 3,45 & 3,69 & 2,59 & 3,58 \\
\hline $\mathrm{K}_{2} \mathrm{O}$ & 5,17 & 5,27 & 3,23 & 4,18 & 4,85 & 5,99 & 3,83 \\
\hline $\mathrm{P}_{2}^{2} \mathrm{O}_{5}$ & 0,08 & 0,10 & 0,15 & 0,07 & 0,11 & 0,20 & 0,35 \\
\hline LOI & 0,61 & 0,68 & 0,93 & 0,43 & 0,50 & 0,53 & 1,17 \\
\hline Total & 99,23 & 100,60 & 100,90 & 99,63 & 100,20 & 99,81 & 99,48 \\
\hline $\mathrm{Sc}$ & 3 & 3 & 3 & 4 & 2 & 1 & 5 \\
\hline $\mathrm{Be}$ & 3 & 3 & 2 & 3 & 5 & 2 & 5 \\
\hline V & $<5$ & $<5$ & 18 & 14 & 7 & $<5$ & 26 \\
\hline $\mathrm{Cr}$ & 20 & $<20$ & $<20$ & $<20$ & $<20$ & $<20$ & $<20$ \\
\hline Co & 1 & 1 & 3 & 2 & 1 & 1 & 5 \\
\hline $\mathrm{Ni}$ & $<20$ & $<20$ & $<20$ & $<20$ & $<20$ & $<20$ & $<20$ \\
\hline $\mathrm{Cu}$ & $<10$ & 30 & 30 & $<10$ & 20 & 420 & $<10$ \\
\hline $\mathrm{Zn}$ & 40 & 40 & 80 & 60 & 50 & 50 & 100 \\
\hline $\mathrm{Ga}$ & 19 & 20 & 23 & 24 & 22 & 17 & 24 \\
\hline $\mathrm{Ge}$ & 1,1 & 1,1 & 0,8 & 1,2 & 1,5 & 0,9 & 1,1 \\
\hline As & $<5$ & $<5$ & $<5$ & $<5$ & $<5$ & $<5$ & $<5$ \\
\hline $\mathrm{Rb}$ & 248 & 256 & 227 & 227 & 262 & 268 & 235 \\
\hline $\mathrm{Sr}$ & 115 & 111 & 247 & 227 & 134 & 112 & 207 \\
\hline Y & 6,9 & 6,4 & 12,5 & 12,6 & 11,8 & 9,8 & 13,4 \\
\hline $\mathrm{Zr}$ & 107 & 91 & 262 & 166 & 111 & 127 & 179 \\
\hline $\mathrm{Nb}$ & 7,6 & 7,5 & 12,0 & 16,8 & 12,4 & 7,5 & 17,7 \\
\hline Мо & $<2$ & $<2$ & $<2$ & $<2$ & $<2$ & $<2$ & $<2$ \\
\hline $\mathrm{Ag}$ & $<0,5$ & $<0,5$ & $<0,5$ & $<0,5$ & $<0,5$ & 2,40 & $<0,5$ \\
\hline In & $<0,1$ & $<0,1$ & $<0,1$ & $<0,1$ & $<0,1$ & $<0,1$ & $<0,1$ \\
\hline Sn & 1 & 1 & 3 & 4 & 4 & 1 & 4 \\
\hline Cs & 2,1 & 2,1 & 2,6 & 2,7 & 7,4 & 1,9 & 6,6 \\
\hline $\mathrm{Ba}$ & 768 & 670 & 1.668 & 1.246 & 742 & 905 & 1.072 \\
\hline $\mathrm{Hf}$ & 3,7 & 3,3 & 6,9 & 4,8 & 3,4 & 4,1 & 5,0 \\
\hline $\mathrm{Ta}$ & 1,13 & 1,04 & 0,86 & 1,95 & 2,00 & 0,80 & 1,77 \\
\hline W & 0,5 & 0,6 & $<0,5$ & $<0,5$ & $<0,5$ & $<0,5$ & $<0,5$ \\
\hline $\mathrm{Pb}$ & 49 & 70 & 31 & 31 & 29 & 42 & 26 \\
\hline $\mathrm{Bi}$ & 3,8 & 3,7 & 0,2 & $<0,1$ & 0,4 & 1,1 & 0,5 \\
\hline Th & 27,4 & 24,4 & 51 & 33,6 & 27 & 25,1 & 24,6 \\
\hline $\mathrm{U}$ & 4,7 & 3,3 & 5,2 & 3,2 & 5,9 & 3,3 & 3,4 \\
\hline
\end{tabular}

Tabla 2.-Análisis de tierras raras (ppm) del granito de Banabuiú

\begin{tabular}{|c|c|c|c|c|c|c|c|}
\hline & MI-01 & MI-184 & MI-93A & MI-206 & MI-212 & MI-214 & MI-105G \\
\hline $\mathrm{La}$ & 43,50 & 37,20 & 125,00 & 79,90 & 45,50 & 50,50 & 65,80 \\
\hline $\mathrm{Ce}$ & 83,20 & 73,20 & 211,00 & 144,00 & 76,50 & 100,00 & 124,00 \\
\hline $\mathrm{Pr}$ & 8,21 & 7,36 & 18,70 & 12,60 & 7,01 & 8,87 & 11,10 \\
\hline $\mathrm{Nd}$ & 25,20 & 23,20 & 51,60 & 37,50 & 22,60 & 30,00 & 34,70 \\
\hline $\mathrm{Sm}$ & 4,52 & 4,20 & 7,99 & 6,24 & 4,04 & 5,65 & 6,41 \\
\hline $\mathrm{Eu}$ & 0,59 & 0,54 & 1,05 & 0,97 & 0,54 & 0,55 & 0,92 \\
\hline Gd & 1,53 & 1,71 & 4,56 & 3,82 & 3,11 & 3,75 & 4,17 \\
\hline $\mathrm{Tb}$ & 0,24 & 0,23 & 0,51 & 0,50 & 0,42 & 0,46 & 0,54 \\
\hline Dy & 1,24 & 1,13 & 2,26 & 2,34 & 2,02 & 2,04 & 2,48 \\
\hline Ho & 0,19 & 0,17 & 0,42 & 0,41 & 0,36 & 0,33 & 0,42 \\
\hline $\mathrm{Er}$ & 0,40 & 0,38 & 1,23 & 1,11 & 1,01 & 0,85 & 1,16 \\
\hline Tm & 0,05 & 0,05 & 0,18 & 0,15 & 0,15 & 0,11 & 0,16 \\
\hline $\mathrm{Yb}$ & 0,27 & 0,27 & 1,20 & 0,93 & 0,95 & 0,67 & 1,03 \\
\hline $\mathrm{Lu}$ & 0,03 & 0,03 & 0,19 & 0,13 & 0,12 & 0,09 & 0,16 \\
\hline Total & 169,17 & 149,67 & 425,89 & 290,60 & 164,33 & 203,87 & 253,04 \\
\hline
\end{tabular}



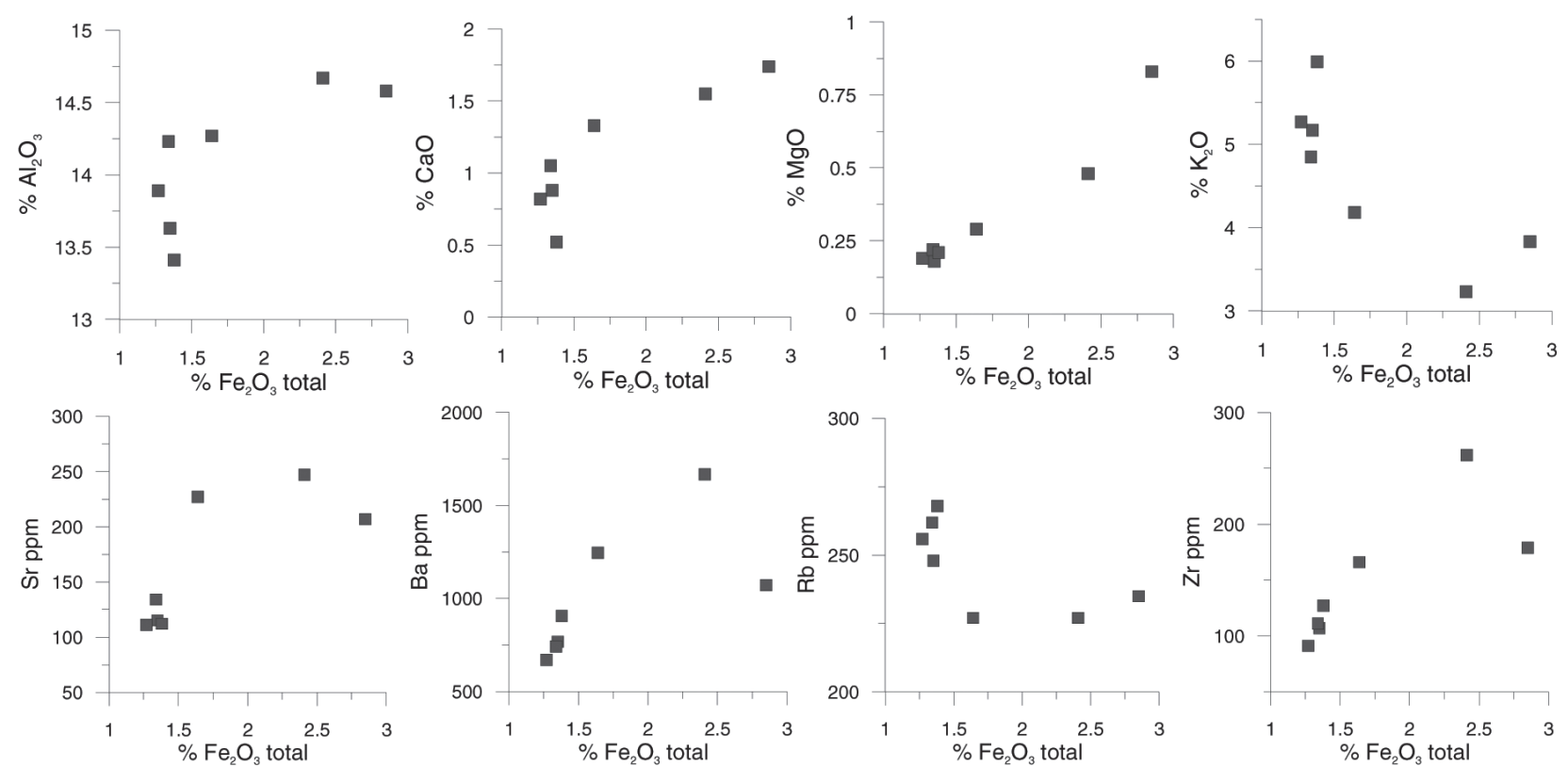

Fig. 6.-Diagramas de variación de elementos mayores y traza en el granito de Banabuiú.

regular del aluminio. En conjunto, las relaciones observadas muestran que la evolución de estos magmas estuvo controlada por procesos de cristalización fraccionada, envolviendo biotita, ilmenita, plagioclasa y apatito \pm monacita. La disminución sistemática de $\mathrm{Sr}, \mathrm{Ba}, \mathrm{U}, \mathrm{Th}, \mathrm{Y}, \mathrm{Nb}, \mathrm{Zr}, \mathrm{Hf}, \mathrm{La}$ y $\Sigma T R$ a medida que tiene lugar la diferenciación (fig. 6) es compatible con la movilización por cristalización fraccionada de una asociación mineralógica constituida por plagioclasa $(\mathrm{Sr})+$ biotita $(\mathrm{Ba}$, $\mathrm{Nb})+$ apatito/monacita (La, $\Sigma \mathrm{TR} ; \mathrm{Th}, \mathrm{Y})+$ circón (Zr, Hf, U, Th) (fig. 6).

Los diagramas de tierras raras de las muestras del granito de Banabuiú se caracterizan por (fig. 7): a) disminución de las tierras raras leves (TRL) con el aumento de la diferenciación; $b$ ) enriquecimiento de las TRL con respecto a las tierras raras pesadas (TRP) $\left(\mathrm{La}_{\mathrm{N}} / \mathrm{Yb}_{\mathrm{N}}=32,33-108,75\right)$; c) moderado fraccionamiento de las TRP $\left(\mathrm{Gd}_{N} / \mathrm{Yb}_{\mathrm{N}}=2,65-5,12\right)$, y d) presencia de anomalías negativas de Eu de amplitud variable $\left(\mathrm{Eu} / \mathrm{Eu}^{*}=0,34-0,56\right)$. El paralelismo de los perfiles es elevado, lo que indica que los diferentes términos de la secuencia derivan del mismo magma parental. La mayor parte de las muestras presenta anomalías negativas de Eu bien definidas, demostrando el papel determinante del fraccionamiento de la plagioclasa en la diversificación magmática. No obstante, dos de las muestras más diferenciadas poseen valores $\mathrm{Eu} / \mathrm{Eu}^{*}$ elevados,

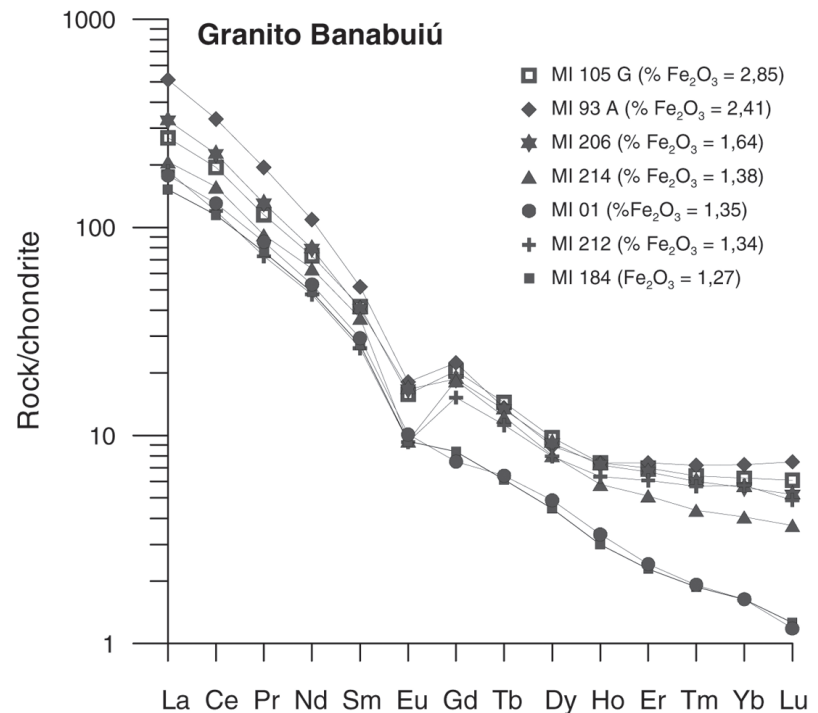

Fig. 7.-Perfiles de tierras raras en las muestras del granito de Banabuiú. Valores normalizados a condrito (Evensen et al., 1978).

reflejando probablemente los efectos de alguna acumulación de feldespato. Por otro lado, la disminución de TRL con la diferenciación, así como el fraccionamiento TRL/TRP, sugiere la participación de apatito y monacita en la asociación mineralógica cuya separación condicionó el paso de los términos menos diferenciados a los más diferenciados. 

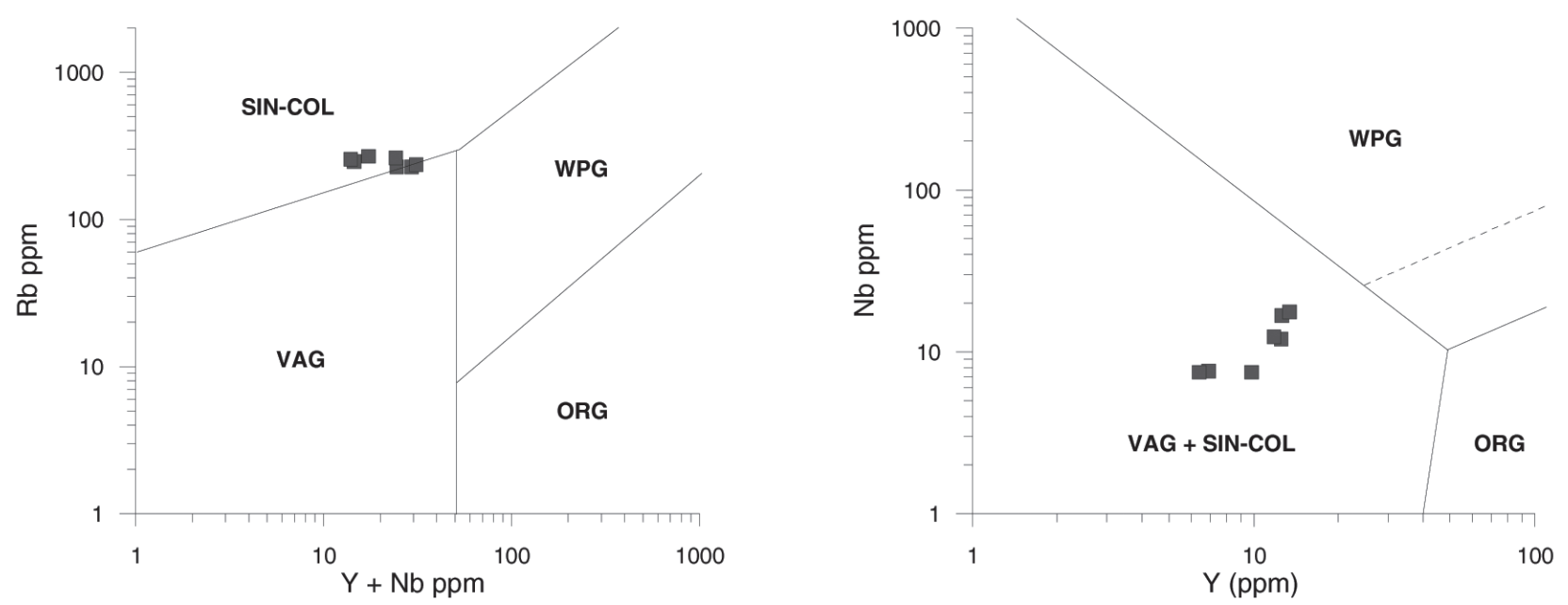

Fig. 8.-Proyección de las muestras del granito de Banabuiú en los diagramas de discriminación tectónica (Pearce et al., 1984). ORG: granitoides de corteza oceánica; SIN-COL: granitoides sin-colisionales; SIN-COL + VAG: granitoides sin-colisionales de arco volcánico; VAG: granitoides de arco volcánico; WPG: granitoides intraplaca.

En los diagramas de discriminación tectónica propuestos por Pearce et al. (1984), el granito de Banabuiú se proyecta en el campo de los granitoides sin-colisionales (SIN-COL) y sin-colisionales de arco volcánico (SIN-COL + VAG) (fig. 8).

\section{Geología isotópica}

Los análisis isotópicos $\mathrm{U}-\mathrm{Pb}$ obtenidos en circones del granito de Banauiú, en el Laboratorio de Geologia Isotópica de la Universidad de Brasília (Brasil), permiten situar la edad del emplazamiento en los 578,6 $\pm 6,5 \mathrm{Ma}(\mathrm{MSWD}=1,7)$ (fig. 9).

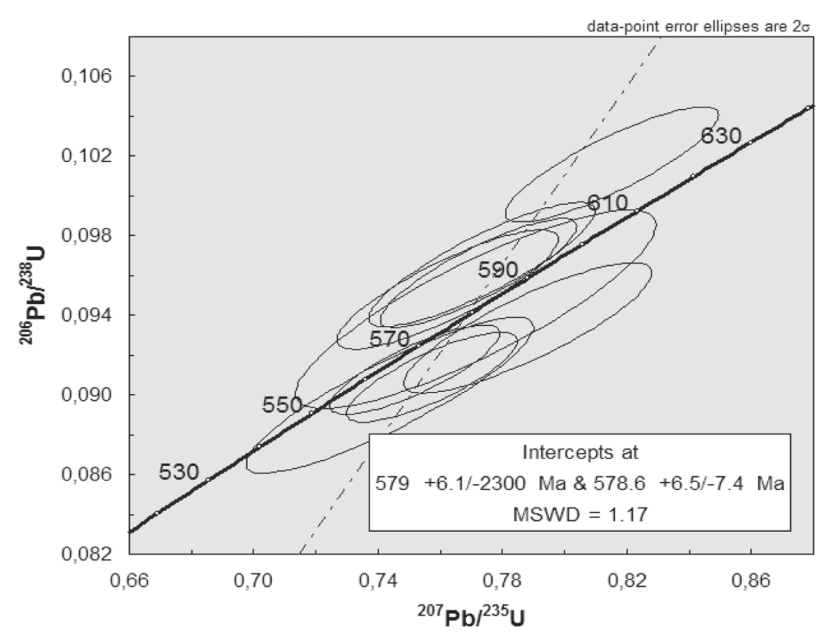

Fig. 9.-Edades U-Pb en circones del granito de Banabuiú.
Esta edad, aunque ligeramente más reciente que la propuesta por Arthaud (2005) y Arthaud et al. (2008) para los granitos sin-brasilienses de tipo-S, es concordante, teniendo en cuenta los márgenes de error, con las de otros batolitos presentes en la región: Quixadá (585 $\pm 4,7 \mathrm{Ma})$ y Quixeramobim (587 $\pm 14 \mathrm{Ma})$ (Nogueira, 2004). Braumgartner et al. (2006) refieren edades semejantes a las obtenidas en este trabajo para granitoides, con composición análoga, de la región de Parelhas, Estado de Rio Grande do Norte.

Por otro lado, la presencia de circones zonados en el granito de Banabuiú, con núcleos de edades paleoproterozoicas corrobora la idea de que estos granitos han sido originados a través de la anatexia cortical de materiales paleoproterozoicos, semejantes a los que se encuentran en el Complejo Gnéisico Migmatítico del DCC y confirma su filiación tipo-S (Lima, 2008).

Con base en los datos isotópicos $\mathrm{Rb}-\mathrm{Sr}$ y $\mathrm{Sm}-\mathrm{Nd}$ adquiridos en muestras del granito y de los gneises migmatíticos del DCC, se han calculado las razones ${ }^{87} \mathrm{Sr}^{86}{ }^{86} \mathrm{Sr}_{(580)}$ y ${ }^{143} \mathrm{Nd} /{ }^{144} \mathrm{Nd}_{(580)}$ así como los respectivos valores de $\varepsilon \operatorname{Sr}_{(580)}$ y $\varepsilon \mathrm{Nd}_{(580)}$ (tabla 3 ). $\mathrm{El} \varepsilon \mathrm{Nd}_{(580)}$ presenta valores negativos muy acusados y se sobreponen parcialmente a los del Complejo Gneisico Migmatítico del DCC $\left(\varepsilon \mathrm{Nd}_{580}=-12 \mathrm{y}-26\right)$, sugiriendo que el magma parental del granito de Banabuiú podría haber derivado de la fusión parcial de estos materiales. En contraste, las razones ${ }^{87} \mathrm{Sr} /{ }^{86} \mathrm{Sr}_{(580)}$ son más heterogéneas, debido posiblemente a la perturbación isotópica del sistema $\mathrm{Rb}-\mathrm{Sr}$ 
Tabla 3.-Análisis isotópicos Rb-Sr y Sm-Nd del granito de Banabuiú

\begin{tabular}{llcccrc}
\hline Muestras & ppm Sr & ppm Rb & ${ }^{87} \mathrm{Rb} /{ }^{86} \mathrm{Sr}$ & ${ }^{87} \mathrm{Sr} /{ }^{86} \mathrm{Sr}$ & ${ }^{\varepsilon{ }_{580}}$ \\
\hline MI 93A & 247 & 227 & 2,66 & $0,729933 \pm 3$ & 57,8 \\
MI 214 & 112 & 268 & 6,97 & $0,779440 \pm 3$ & 255,2 \\
MI 01 & 113,76 & 257,75 & 6,59 & $0,767471 \pm 4$ & 129,6 \\
MI 184 & 109,89 & 265,89 & 7,04 & $0,771128 \pm 4$ & 128,6 \\
\hline Muestras & ppm Nd & ppm Sm & ${ }^{147} \mathrm{Sm} /{ }^{144} \mathrm{Nd}$ & ${ }^{143} \mathrm{Nd} /{ }^{144} \mathrm{Nd}$ & $\varepsilon^{2} \mathrm{Nd}_{580}$ & \multicolumn{2}{c}{$\mathrm{T}_{\mathrm{DM}}$} \\
\hline MI 93A & 51,6 & 7,99 & 0,09 & $0,511058 \pm 2$ & $-23,2$ & $2,43 \mathrm{Ga}$ \\
MI 214 & 30 & 5,65 & 0,11 & $0,511269 \pm 2$ & $-20,6$ & $2,59 \mathrm{Ga}$ \\
MI 01 & 25,2 & 4,52 & 0,11 & $0,511282 \pm 2$ & $-19,9$ & $2,45 \mathrm{Ga}$ \\
MI 184 & 23,2 & 4,2 & 0,11 & $0,511304 \pm 2$ & $-199,6$ & $2,44 \mathrm{Ga}$ \\
\hline
\end{tabular}

* Los análisis isotópicos Rb-Sr y Sm-Nd fueron realizados en el Laboratorio de Geología Isotópica de la Universidad de Aveiro, Portugal (LGI-UA).

por procesos de fusión en desequilibrio y/o por alteración hidrotermal tardía. Las edades modelo del granito $\left(\mathrm{T}_{\mathrm{DM}}=2,43\right.$ a $\left.2,59 \mathrm{Ga}\right)$ confirman la hipótesis de un origen a partir de fuentes corticales con un tiempo de residencia largo, tales como las rocas paleoproterozoicas que constituyen el encajante de la región estudiada (Lima, 2008).

\section{Conclusiones}

El macizo de Banabuiú está constituido por un monzogranito de dos micas cuyo emplazamiento fue sin-cinemático tardío con respecto a la deformación orogénica brasiliense, en el Neoproterozoico. Sus características petrográficas, geoquímicas e isotópicas son compatibles con una filiación de tipo-S. Por otro lado, las correlaciones entre la mayor parte de los elementos mayores y trazas $\left(\mathrm{MgO}, \mathrm{TiO}_{2}, \mathrm{MnO}, \mathrm{CaO}, \mathrm{Na}_{2} \mathrm{O}, \mathrm{P}_{2} \mathrm{O}_{5}, \mathrm{Sr}, \mathrm{Ba}, \mathrm{U}\right.$, $\mathrm{Th}, \mathrm{Y}, \mathrm{Nb}, \mathrm{Zr}, \mathrm{Hf}$, La y $\Sigma \mathrm{TR}$ ) y el índice de diferenciación magmática muestran que la evolución del magma estuvo controlada por procesos de cristalización fraccionada, envolviendo una asociación mineralógica formada por plagioclasa + biotita + apatito/monacita + circón. Los análisis isotópicos $\mathrm{U}-\mathrm{Pb}$ obtenidos en circones del granito de Banabuiú dan una edad de emplazamiento de 578,6 \pm 6,5 Ma, confirmando su inclusión en el grupo de los granitoides sin-colisionales. Tanto los valores de $\varepsilon \mathrm{Nd}_{(580)}$ como los de las edades modelo $\left(\mathrm{T}_{\mathrm{DM}}\right)$ del granito sugieren una génesis por fusión parcial a gran escala de protolitos corticales, semejantes a los que se encuentran en el encajante metamórfico de la región estudiada.

\section{AGRADECIMENTOS}

Este trabajo se ha beneficiado del apoyo del Conselho Nacional de Desenvolvimento Científico e Tecnológico (CNPq) a través del proyecto titulado «Período de alojamento e relações petrogenéticas entre pegmatitos e rochas encaixantes da região de Banabuiú-Solonópole (CE), sub-província pegmatítica do Ceará», Proceso nº 478313/2006-3 y de una beca concedida por el Programa Alban (EO6M103314BR). Los autores agradecen también al Laboratorio de Geología Isotópica de la Universidad de Brasília por el apoyo concedido para la obtención de los datos isotópicos.

\section{Referencias}

Almeida, F.F.M.; Melcher, G.C.; Cordani, U.G.; Kawashita, K. \& Vandoros, P. (1968). Radiometric age determinations from northern Brazil. Boletim da Sociedade Brasileira de Geologia, 18: 3-14.

Arthaud, M.H.; Vasconcelos, A.M.; Nogueira Neto, J.A.; Oliveira, F.V.C.; Parente, C.V.; Monié, P.; Liégeois, J.P.; Caby, R. \& Fetter, A.H. (1998). Main Structural Features of Precambrian Domains From Ceará (NE Brazil). International Conference on Basement Tectonics, 14th, Ouro Preto, Abstracts: 84-85.

Arthaud, M.H. (2005). Geologia e tectônica no Domínio Ceará Central. Qualificação ao Doutorado em Geologia, Programa de Pós-Graduação em Geologia, Instituto de Geociências, Universidade de Brasília (UNB), $270 \mathrm{pp}$.

Arthaud, M.H.; Caby, R.; Fuck, R.A.; Dantas, E.L. \& Parente, C.V. (2008). Geology of the northern Borborema Province, NE Brazil and its correlation with Nigeria, NW Africa. In: West Gondwana: Pre-Cenozoic Correlations Across the South Atlantic Region (Pankhurst, R.J., Trouw, R.A.J., Brito Neves, B.B. \& De Wit M.J., eds.). Geological Society, London, Special Publications, 294: 49-67. 
Barker, F. (1979). Trondhjemite: definition, environment and hypotheses of origin. In: Trondhjemites, Dacites and Related Rocks (F. Barker, ed.), Developments in Petrology 6, Elsevier, Amsterdam, 1-12.

Baumgartner, R.; Rolf, L.; Romer, R.L.; Moritz, R.; Sallet, R. \& Chiaradia, M. (2006). Columbite-tantalitebearing granitic pegmatites from the Seridó Belt, NE Brazil: genetic constraints from $\mathrm{U}-\mathrm{Pb}$ dating and $\mathrm{Pb}$ isotopes. Canadian Mineralogist, 44: 69-86. doi:10.2113/gscanmin.44.1.69

Brito Neves, B.B.; Santos, E.J. \& Van Schmus, W.R., (2000). Tectonic History of the Borborema Province, Northeastern Brazil, Proceedings of the $31^{\text {st }}$ International Congress, Rio de Janeiro: 151-182.

Delgado, I.M.; Souza, J.D.; Silva, L.C.; Silveira Filho, N.C.; Santos, R.A.; Pedreira, A.J.; Guimarães, J.T.; Angelim, L.A.A.; Vasconcelos, A.M.; Gomes, I.P.; Lacerda Filho, J.V.; Valente, R.C.; Perrota, M.M. \& E Heineck, C.A. (2003). Geotectônica do Escudo Atlântico. In: Geologia, Tectônica e Recursos Minerais do Brasil (Bizzi, L.A.; Schobbenhaus, C.; Vidotti, R.M. \& Gonçalves, J.H., eds.). CPRM, Brasília, Brasil, 227-334.

Evensen, N.H.; Hamilton, P.J. \& O'Nions, R.K. (1978). Rare earth abundances in chondrite meteorites. Geochimica et Cosmochimica Acta, 42: 1199-1212. doi:10.1016/0016-7037(78)90114-X

Fetter, A.H. (1999). U/Pb and Sm/Nd Geochronological Constraints On The Crustal Framework and Geologic History Of Ceará State, NW Borborema Province, NE Brazil: Implications For The Assembly Of Gondwana. PhD Thesis, Department of Geology, Kansas University, Lawrence, 164 pp.

Fetter, A.H.; Van Schmus, W.R.; Santos, T.J.S.; Nogueira Neto, J.A. \& Arthaud, M.H. (2000). U/Pb and Sm/Nd geochronological constraints on the crustal evolution and basement architecture of Ceará State, NW Borborema Province, NE Brazil: Implications for the txistence of the paleoproterozoic supercontinent «Atlantica». São Paulo. Revista Brasileira de Geociências, 30: 102-106.

Foster, M.D. (1960). Interpretation of trioctahedrical micas. U.S. Geological Survey Professional Paper, 354-B: $1-49$.
Le Bas, M.J. \& Streckeisen, A.L. (1991). The IUGS systematics of igneous rocks. Journal of the Geological Society of London, 148: 825-833. doi:10.1144/gsjgs.148.5.0825

Lima, M.N. (2008). Caracterização litogeoquímica do granito Banabuiú e seu encaixante (Brasil). Tesis de Mestrado. Universidade de Aveiro, Portugal, 168 pp.

Mattos, I.C.; Artur, A.C.; Nogueira Neto, J.A. (2003). Caracterização físico-mecânica do Granito Ornamental Serra do Barriga, Sobral/CE: resultados preliminares. Anais do IV Simpósio de Rochas Ornamentais do Nordeste, Rio de Janeiro, CETEM/SBG, 1: 86-93.

Nachit, H.; Razafimahefa; N.; Stussi, J.M. \& Carron, J.P. (1985). Composition chimique des biotites et typologie magmatique des granitoïdes. Comptes Rendus de l'Academie dês Sciences de Paris, 301: 813-818.

Nockolds, S.R. (1947). The relation between chemical composition and paragenesis in the biotite micas of igneous rocks. American Journal of Science, 245: 401-420.

Nogueira, J.F. (2004). Estrutura, Geocronologia e Alojamento dos Batólitos de Quixadá, Quixeramobim e Senador Pompeu - Ceará Central. Tesis Doctoral. Universidade Estadual Paulista, Rio Claro, São Paulo, $123 \mathrm{pp}$.

Pearce, J.; Harris, N. \& Tindle, A. (1984). Trace element discrimination diagrams for the tectonic interpretation of granitic rocks. Journal of Petrology, 25: 956-983.

Tavares Jr., S.S., (1992). Caracterização litoquímica e geocrolologia $\mathrm{Rb} / \mathrm{Sr}$ das rochas granitóides e ortognaisses da região de Santa Quitéria-Sobral, NW do Ceará. Tesis de Mestrado. Curso de Pós-Graduação em Geociências, Universidade Federal do Pará, 143 pp.

Van Schmus, W.R.; Brito Neves, B.B.; Hackspacher, P.; Fetter, A.H.; Kozuch, M.; Dantas, E.L. \& Babinski, M. (1997). The Borborema Province: A collage of polycyclic crustal domains in NE Brazil. Livro de resumos do XVII Simpósio de Geologia do Nordeste, 115-120.

Recibido el 18 de enero de 2010 Aceptado el 7 de abril de 2010 Publicado online el 9 de junio de 2010 